\title{
The Structure of Scientific Knowledge
}

\author{
Charles W Misner
}

University of Maryland 


\section{Provocation}

- Problem: "It's just a theory"

- Excuse: Popper and Kuhn --

Current theory could be falsified;

The better theory could bring in

new objects and actions.

- Solution: Use only "Certified Theories" (which have already been shown false) in the domains where they are certified. 


\section{Solution}

- Define an Certified Theory as one which has

- Known limits

- Within which it is certified by better theories as correct and reliable

- Is in active use by scientists who understand the theories which replace it at its limits,,,

- And which, in its proper domain, is more insightful than the more cumbersome theories which define its limits. 


\section{Challenge and Detour}

- But there are many certified theories, and they are defined by their relationships to other theories

- So we need to study relationships between theories

- That occurs within a wide ranging survey of what scientific knowledge can mean.

- Scientific aims

- Scientific accomplishments 
- Pure Science vs. Applied

- Basic vs. Derived (cf. Chandra)

- Normal or Paradigm Shift (cf. Kuhn)

- Reductionist vs. Emergent (cf. Anderson)

- Accepted vs. Falsified (cf. Popper)

- Cutting Edge vs. Certified 


\section{physics Basic vs. Derived Science}

- Basic discoveries

- Maxwell's equations

- Dirac equation

- QCD, etc.

- Dark matter

- Derived discoveries

- Chadrasekhar limit for white dwarfs

- positrons

- Black Holes 


\section{Normal or Paradigm Shift}

- Normal science (correct the details)

- Dark Energy (in GR normal, not in QFT)

- Dark Matter (no change to Einstein's equations needed)

- Allow for small neutrino masses

- Paradigm Shift

- Go from 4D to 11D?

- Æther to Special Relativity Vacuum

- Fixed spacetime to dynamically curved spacetime

- Empty vacuum to quantum virtual particle æther 
- Recent success "Dark Matter Telescope"

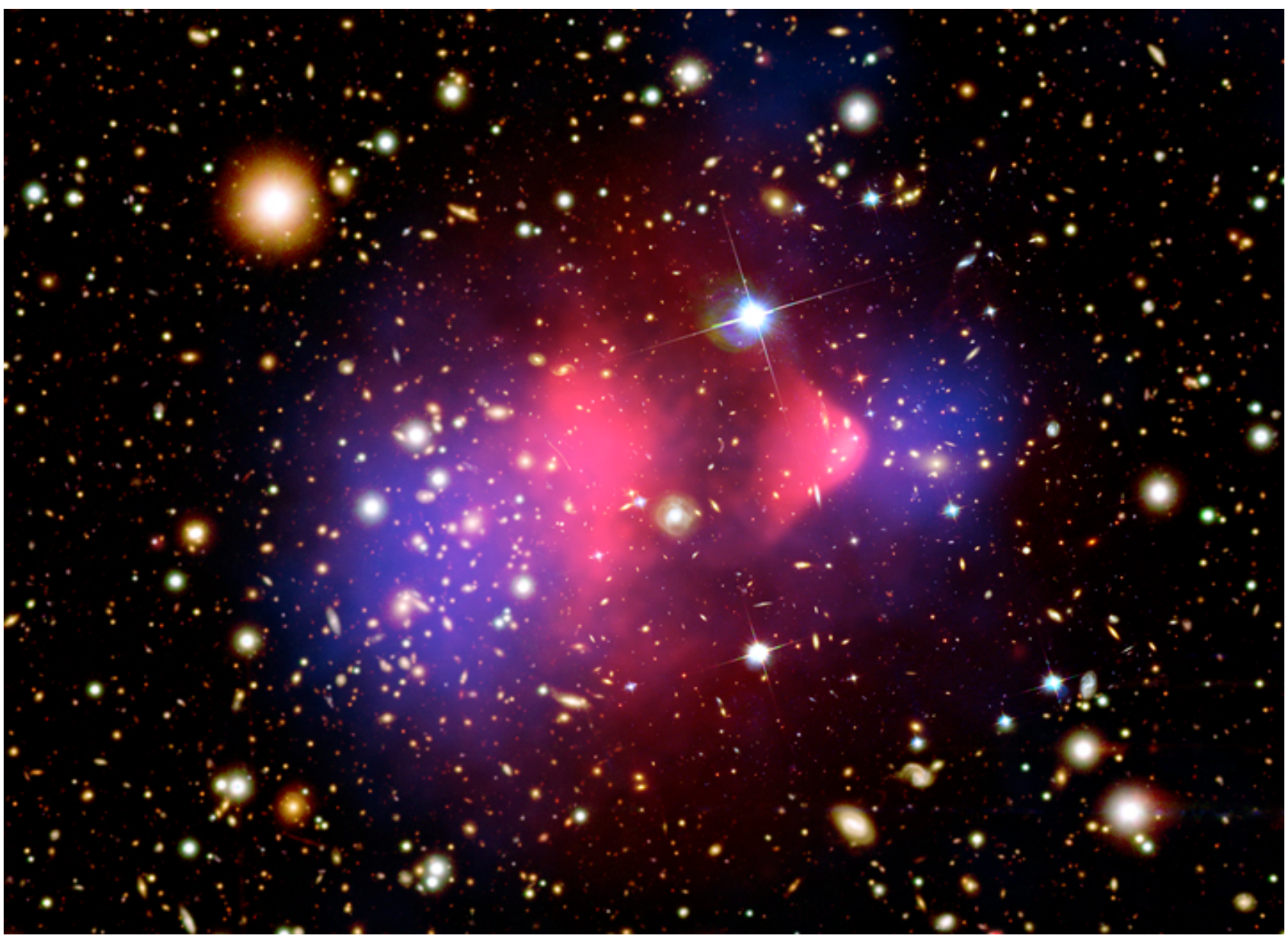


- Pure Science vs. Applied $J$

- Basic vs. Derived (cf. Chandra) J

- Normal or Paradigm Shift (cf. Kuhn) J

- Reductionist vs. Emergent (cf. Anderson)

- Accepted vs. Falsified (cf. Popper)

- Cutting Edge vs. Certified 


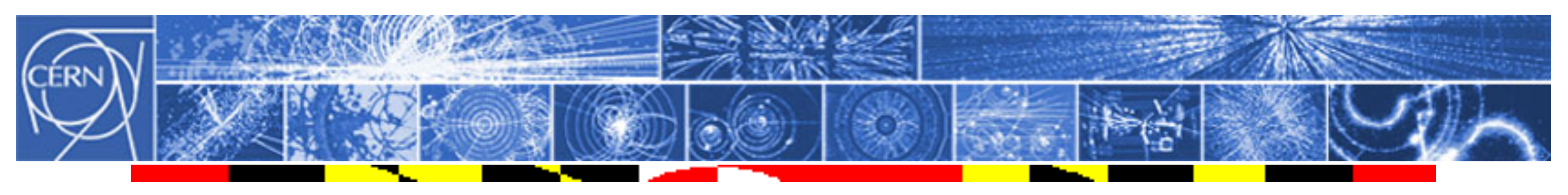

\section{After more than 50 years of experiments our present understanding of the Universe}
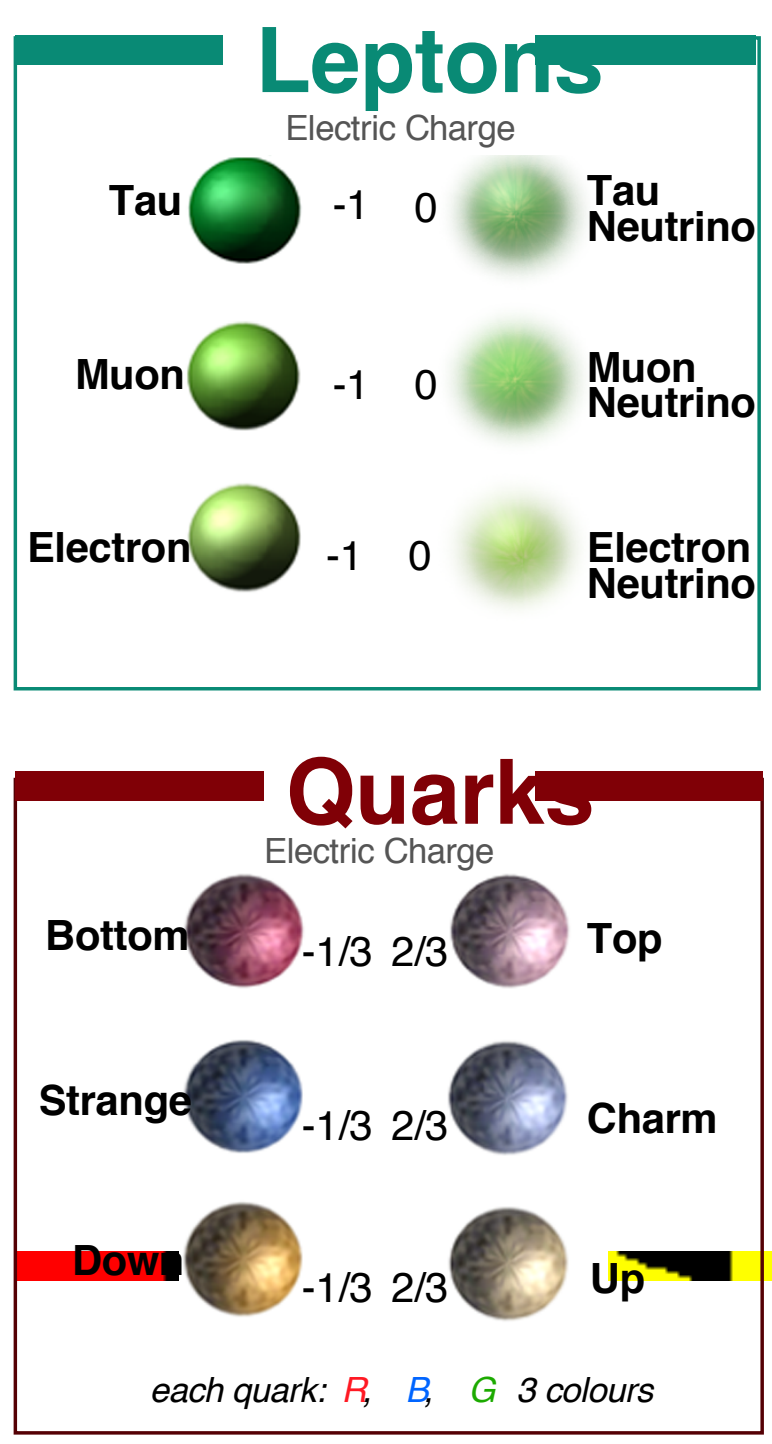
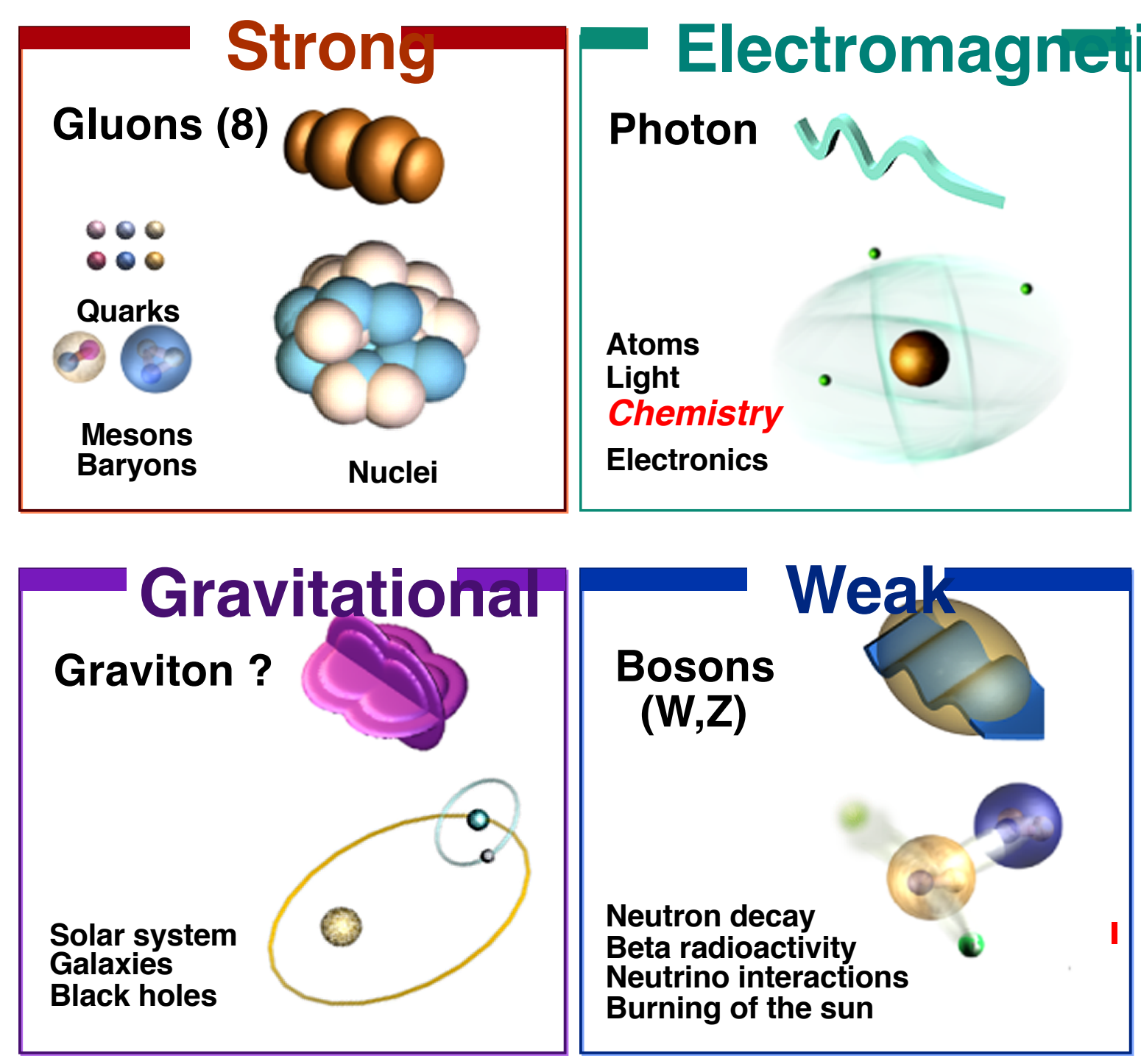


\section{Reductionist Chemistry}

Equation to Solve in ab initio Theory

\section{$H \Psi=\mathrm{E} \Psi$}

Known exactly:

$3 N$ spatial variables

( $N$ \# of electrons)
To be approximated:

1. variationally

2. perturbationally 


\section{Reductionist Chemistry}

\section{Hamiltonian for a Molecule}

$$
\begin{aligned}
\hat{\mathbf{H}}= & \sum_{i}^{\text {electrons }} \frac{-\hbar^{2}}{2 m_{e}} \nabla_{i}^{2}+\sum_{A}^{\text {nuclei }} \frac{-\hbar^{2}}{2 m_{A}} \nabla_{A}^{2}+\sum_{i}^{\text {electronsnuclei }} \sum_{A} \frac{-e^{2} Z_{A}}{r_{i A}} \\
& +\sum_{i>j}^{\text {electrons }} \frac{e^{2}}{r_{i j}}+\sum_{A>B}^{\text {nuclei }} \frac{e^{2} Z_{A} Z_{B}}{R_{A B}}
\end{aligned}
$$

- kinetic energy of the electrons

- kinetic energy of the nuclei

- electrostatic interaction between the electrons and the nuclei

- electrostatic interaction between the electrons

- electrostatic interaction between the nuclei 


\section{Reductionist Example}

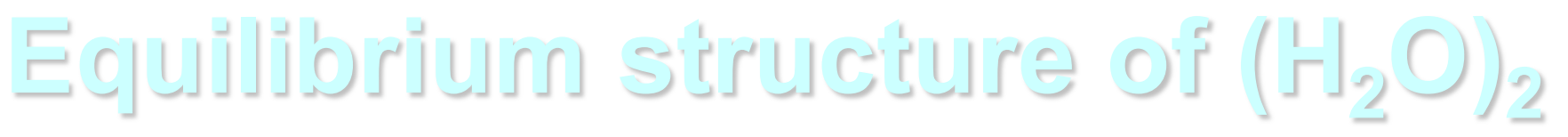

W.K., J.G.C.M. van Duijneveldt-van de Rijdt, and F.B. van Duijneveldt, Phys. Chem. Chem. Phys. 2, 2227 (2000).

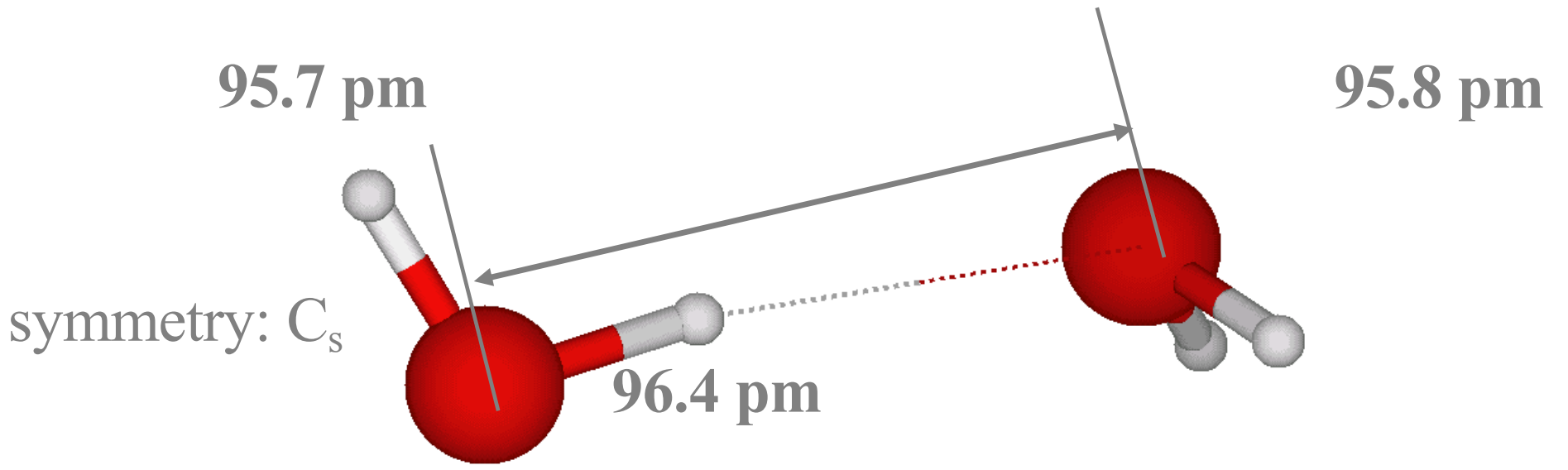

Experimental [J.A. Odutola and T.R. Dyke, J. Chem. Phys 72, 5062 (1980)]:

$$
\left.<\boldsymbol{R}_{\mathrm{OO}^{-2}}\right\rangle^{-1 / 2}=297.6 \pm 0.4 \mathrm{pm}
$$

SAPT-5s potential [E.M. Mas et al., J. Chem. Phys. 113, 6687 (2000)]:

$$
<\boldsymbol{R}_{\mathrm{OO}^{-2}}>^{-1 / 2}-R_{\mathrm{OO}, \mathrm{e}}=6.3 \mathrm{pm} \rightarrow \boldsymbol{R}_{\mathrm{OO}, \mathrm{e}}(\operatorname{exptl} .)=291.3 \mathrm{pm}
$$


physics Emergent Chemistry

From Hanson et al., Science v335, p851, SOM p 22
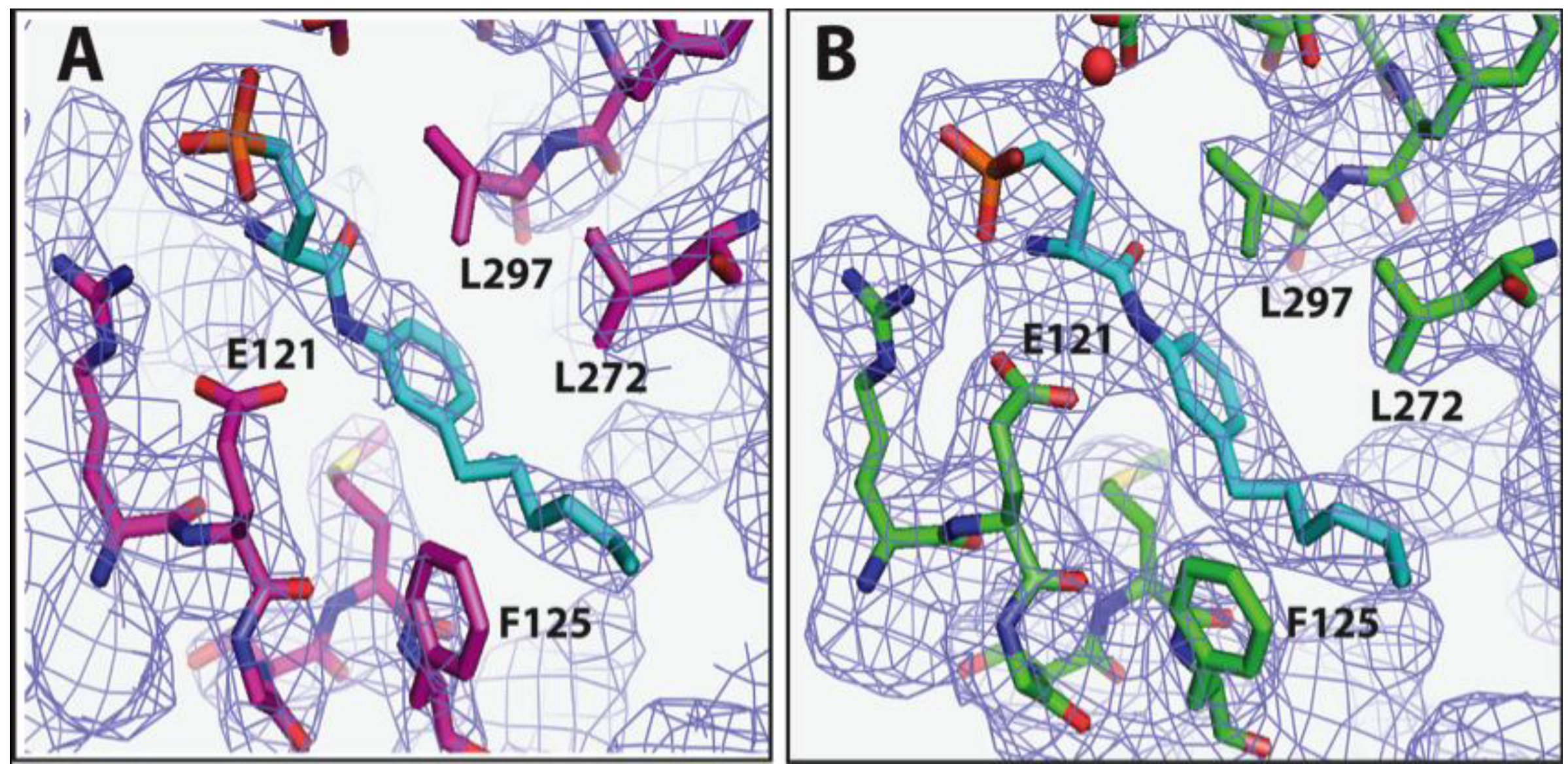


\section{Emergent Chemistry}

In DNA even the atoms, much less individual electrons, are barely noticed.

The base pairs are the basic unit.

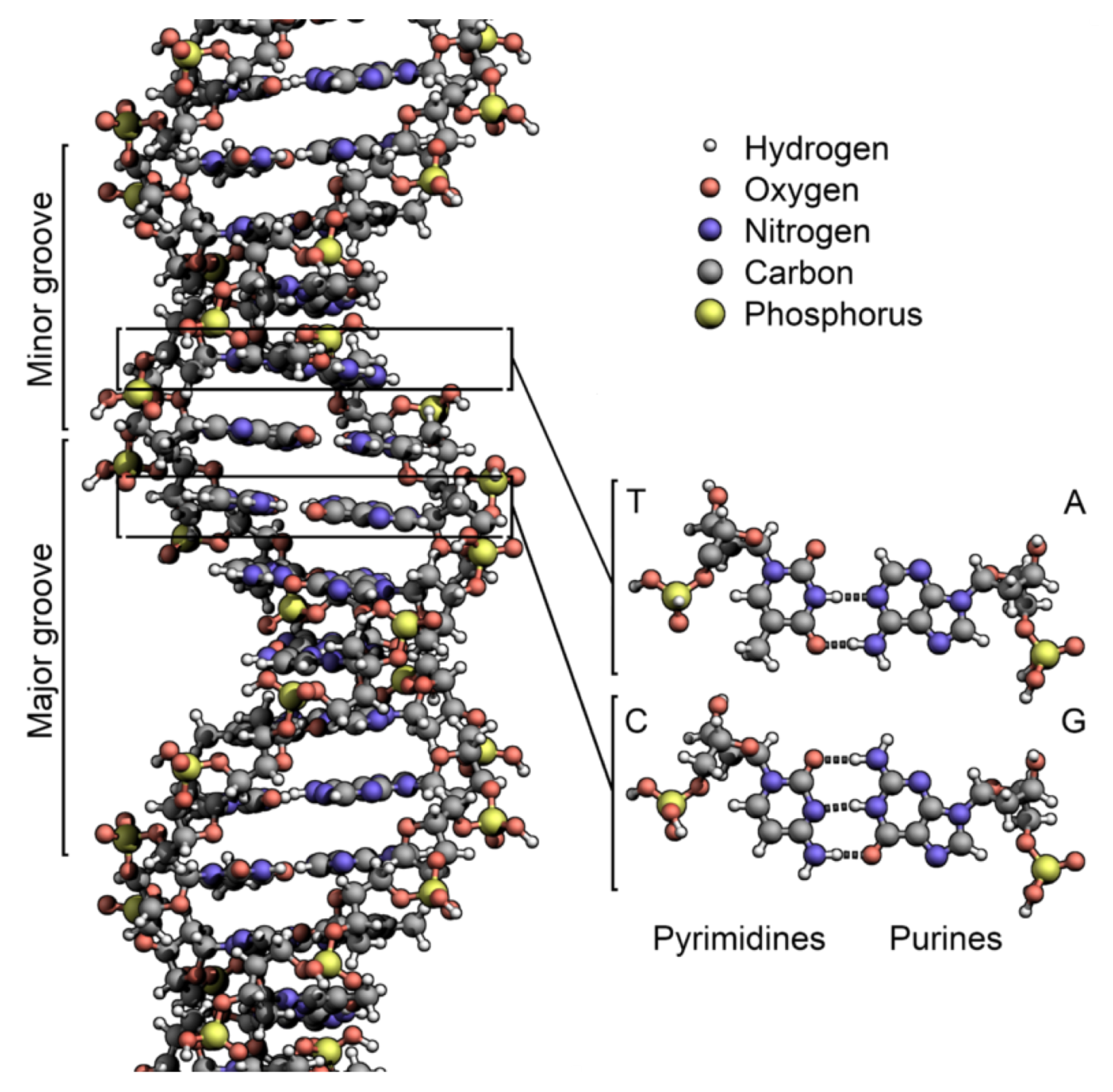




\section{Emergent Chemistry}

- As a simpler example of reductionist vs. emergent concepts in chemistry consider the "More is Different" (Ph. Anderson) view of the Carbon Atom as a building block.

- Is there anything visible in the Schrödinger equation view of the carbon atom that would lead to both

- Diamond, and

- Graphite

as possible quasistable many-particle equilibria? 


\section{Emergent Theories}

- P. W. Anderson, "More is Different", Science, Vol. 177, pp. 393-396 (1972)

- Philip W. Anderson, Chapter 3 in More and Different (World Scientific, 2011) ISBN 978981-4350-13-6 
- Pure Science vs. Applied $J$

- Basic vs. Derived (cf. Chandra) J

- Normal or Paradigm Shift (cf. Kuhn) J

- Reductionist vs. Emergent (cf. Anderson) J

- Accepted vs. Falsified (cf. Popper)

- Cutting Edge vs. Certified 


\section{Scientific Aims}

Agreement with experiment \& observation. But

- Extrapolation is not enough (e.g. tide tables)

- Reductionist theme is prominent

- Explain reality by its underlying constitutents, e.g. "Theory of Everything"

- Needs both basic science and derived science (see Dyson at Chandra100)

- Emergent laws and properties are essential

- Thermodynamics, Hydrodynamics, Chemical bonds, ... (see Philip Anderson) 


\section{Scientific Aims}

- The best current theories are subject to change.

- Ambitious scientists are working to find where they are wrong, which could win them a prize. 


\section{physics Scientific Accomplishments}

- Theories get falsified - Popper

- A statement is not scientific if one can't imagine an experiment of observation that might prove it wrong.

- This has happened previously; why not again?

- Scientific revolutions - Kuhn

- Not only the rules change

- Even the vocabulary/concepts may be entirely novel 


\section{physics Scientific Accomplishments}

The growing lode of permanent knowledge:

- Don't rely on the best current theories - look elsewhere.

- But the trash bin of falsified theories doesn't look inviting at first. Phlogiston anyone?

- But the reusables shop is full of good stuff!

- With Newtonian mechanics as a prime example, we look for "Certified Theories" 
- The core set of Certified Theories with their mutual relationships and their relationships to other theories

- The active troposphere of cutting edge theories, the best we know in any given area

- The stratosphere of speculative theories, representing plausible directions toward better theories. 


\section{Definition}

- Define an Certified Theory as one which has

- Known limits

- Within which it is certified by better theories as correct and reliable

- Is in active use by scientists who understand the theories which replace it at its limits,,,

- And which, in its proper domain, is more insightful than the more cumbersome theories which define its limits. 
- Pre-Ptolemaic motion of celestial objects

- Kepler's laws

- Newtonian Mechanics

- Heisenberg-Schrödinger quantum mechanics

- Maxwell-Lorentz classical electrodynamics

- Schwinger-Feynman-Dyson electrodynamics 


\section{Certified Theories}

A tool needed for recognizing certified theories is the collection of relationships theories may have with each other:

- Correspondence principles

- Emergent laws and concepts

- Evanescent laws and concepts 
- Quantum theory gives classical mechanics when quantum numbers are large

- General Relativity reduces to special relativity when curvature is negligible

- GR reduces to Newtonian gravity for weak fields and slow motions

- Special relativity gives Newtonian mechanics for slow motions

- Electroweak YM gives QED at low energies 


\section{Evanescent Laws}

- A law or property can be called Evanescent when in some domain either

- It becomes irrelevant or useless

- It is indefinable or misleading

- Its normal consequences can be evaded

- It (rarely) is incorrect in some domain

- I could (Oppurg) make the evanescence case for energy and entropy on the cosmological scale. 


\section{Evanescent Laws}

- Examples

- Conservation of QCD color charge as one moves into atomic physics

- Coulomb law as one calculates the motions of solar system planets

- Schrödinger equation in molecular genetics

- Energy on cosmological scales

- Entropy in large self-gravitating systems 
- The expanding lode of reliable scientific knowledge is contained in the core set of Certified Theories

- Some applications of this viewpoint:

- Crackpot theories

- "theory-laden observations" 


\section{Crackpot Theories}

- Defined: a theory which, while explaining (?) some phenomenon, cannot produce by correspondence principles the certified theories which show that centuries of past data make sense. 


\section{"Theory-laden"}

- Some philosophers worry that if we need a bubble chamber to see cosmic rays, that data assumes the theory it is trying to support.

- Worst case: If Archimedes could be resurrected and flown from Syracuse to Geneva then, given full access to CERN, could he see quarks there?

- Response: Even CERN detectors are mainly interpreted using Certified Theories. 


\section{physics Speculation vs. Reliable Sci.}

Projects many scientists find implausible have supporters who treat inconvenient scientific facts as "just a theory"

The viewpoint I present here is provided as background for scientists talking to journalists and politicians.

This presentation here is too technical for direct delivery to a nonscientific public.

It is most applicable in fields with clearly defined theories such as physics, chemistry, genetics. 